\title{
Outbreak investigation in two groups of coach passengers with gastroenteritis returning from Germany to the Netherlands in February 2009
}

H Visser (VisserH2@ggd.rotterdam.nl) ${ }^{1}$, L Verhoef ${ }^{2}$, W Schop ${ }^{1}$, H M Götz ${ }^{1}$

1. Municipal Public Health Service Rotterdam-Rijnmond, Department Infectious Disease Control, Rotterdam, the Netherlands

2. National Institute for Public Health and the Environment (RIVM), Diagnostic Laboratory for Infectious Diseases, Bilthoven, the Netherlands

Citation style for this article:

Visser H, Verhoef L, Schop W, Götz HM. Outbreak investigation in two groups of coach passengers with gastroenteritis returning from Germany to the Netherlands in February 2009. Euro Surveill. 2010;15(28):pii=19615. Available online: http://www.eurosurveillance.org/ViewArticle.aspx?Articleld=19615

In February 2009, an outbreak of 38 cases of gastroenteritis occurred among the participants of two Dutch coach trips (A and B) who visited the same hotel in Germany. We initiated an outbreak investigation to determine possible risk of food-borne infection. A retrospective cohort study was performed among 87 passengers using a self-administered questionnaire. The response rate was 75 of 87 (86\%). Mean age was 65 years. Cases were defined as participants of the two coach trips who had diarrhoea and/or vomiting at least once within 24 hours in the period between 7 and 14 February 2009. We distinguished early and late cases, with symptoms starting within or after 72 hours of arrival in the hotel. Overall attack-rate was 38 of $75(51 \%)$. Microbiological investigation was performed on stool samples of two passengers from Coach A and two passengers from Coach B. Identical norovirus genotype II.4 sequences were detected in all four samples. Univariate analysis revealed a potential risk for early cases from juice consumption, which was most clearly seen for Coach B on day of arrival (juice at lunch: relative risk (RR): 3.9, 95\% confidence interval ( $\mathrm{Cl})$ : 1.3-11.7; juice at dinner: $\mathrm{RR}: 5.5,95 \% \mathrm{Cl}$ : 1.6-18.1). A dose-response relationship was found. This outbreak was probably caused by using the taps of juice served in large containers with a tap for selfservice, due to environmental contamination through person-to-person transmission. Still the role of either contaminated juice or contact with contaminated juice cannot be ruled out.

\section{Introduction}

Noroviruses are a common cause of gastroenteritis outbreaks with an incubation period of 12 to 72 hours. The characteristic symptoms of vomiting and diarrhoea are short-lived, lasting two to three days, but can last longer in older and vulnerable individuals (such as hospitalised persons or residents of nursing homes) [1]. Symptomatic humans can shed the virus as early as several hours before onset of symptoms until three weeks after recovery. Asymptomatic shedding has been reported $[2,3]$. Transmission of the virus can occur through contact with infected persons, contaminated environment or contaminated aerosols, as well as through consumption of contaminated food or water. Given the low dose needed for infection [4] and the fact that person-to-person transmission quickly takes over with high attack rates (30-60\%), identification of a point source of infection during an outbreak is complicated. Moreover, food or water contaminated by noroviruses usually appears to be in good condition and detection of norovirus in food or water is complicated because viruses do not replicate outside their host and are therefore present only in small numbers in the food. Contamination of food can occur at any point during production, preparation and handling of food, including the preparation of individual servings by infected food handlers [5].

On 9 February 2009, the Municipal Public Health Service (MPHS) Rotterdam-Rijnmond was notified of several passengers who had developed symptoms of diarrhoea and/or vomiting within a short time period during a two-day coach trip to Germany. On the evening of 11 February 2009, an outbreak in a second coach was notified, triggering an outbreak investigation. At that time, six passengers were admitted to a hospital in the Netherlands. Both coaches had followed the same 3,5hour travel itinerary, and the two groups of passengers had stayed in the same hotel in Germany for consecutive periods of two days, indicating a potential common source of infection. We investigated the outbreak to determine its size and to identify a potential common food-borne source in order to implement measures limiting further spread to other groups of visitors.

\section{Materials and methods \\ Laboratory analysis}

We obtained stool samples from four patients who were admitted to the emergency ward, two from each coach, and they were tested by norovirus ELISA (RIDASCREEN, R-Biopharm $A G$ ) in a regional diagnostic laboratory. We used the diagnostic algorithm for outbreaks of gastroenteritis used in the Netherlands [6]. The four 
positive samples were sent to the National Institute for Public Health and the Environment (Rijksinstituut voor Volksgezondheid en Milieu, RIVM) for further confirmation of the causative agent using RT-PCR and subsequent sequencing of the capsid gene (VP1) for genotyping and comparison of strains $[7,8]$.

\section{Epidemiological investigation \\ Outbreak description}

Coach A left the Netherlands on 7 February 2009 and returned in the evening of 9 February. Coach $B$ left on 9 February and returned in the evening of 11 February. Itinerary, accommodation and meals served in the hotel were identical for both coach trips. Passengers from Coach A and Coach B were not in contact with each other. The first notification was made to the MPHS Rotterdam-Rijnmond on 9 February 2009.

\section{Data collection}

The MPHS Rotterdam-Rijnmond contacted the organising travel agency, and subsequently the local German public health office (Gesundheitsamt), to obtain the passenger list and information on the meals served in the hotel during the trips. As the menu appeared to be unavailable, we performed hypothesis-generating interviews with six randomly chosen listed passengers and the four passengers whose stool had been investigated, in order to assess the food items served during their stay in the hotel. In addition, the 10 passengers were interviewed using a trawling questionnaire for other details of the journey. Because some of the passengers were resident outside of the RotterdamRijnmond region, an outbreak alert was sent to all MPHSs in the Netherlands. The MPHSs were asked to report any cases linked to the outbreak. We performed a retrospective cohort study among the 87 passengers of both coaches. Demographic data, history of gastroenteritis, consumed food items and possible exposures such as contact with ill persons and which toilets were used during the journey (onboard toilet and toilet use during stops) and during the stay in the hotel (toilet use in the hotel and during excursions) were assessed using self-administered questionnaires.

Data analysis and case definition

The cases were defined as travellers on Coach A or B who had diarrhoea and/or vomiting at least once within 24 hours in the period between 7 and 14 February 2009. To be able to identify a potential common foodborne source of infection, we analysed early and late cases separately in order to distinguish a potential initial source of infection from later person-to-person transmission. Early cases were defined as those with symptom onset within 72 hours after arrival in the hotel, late cases as those with symptom onset later than 72 hours after arrival in the hotel. In a sub-analysis for significant risk factors, we considered a case definition including biological plausibility for a suspected food item or other risk factor to be the source of infection: a biologically plausible case was defined

\section{FIGURE 1}

Steps in the outbreak investigation, February 2009

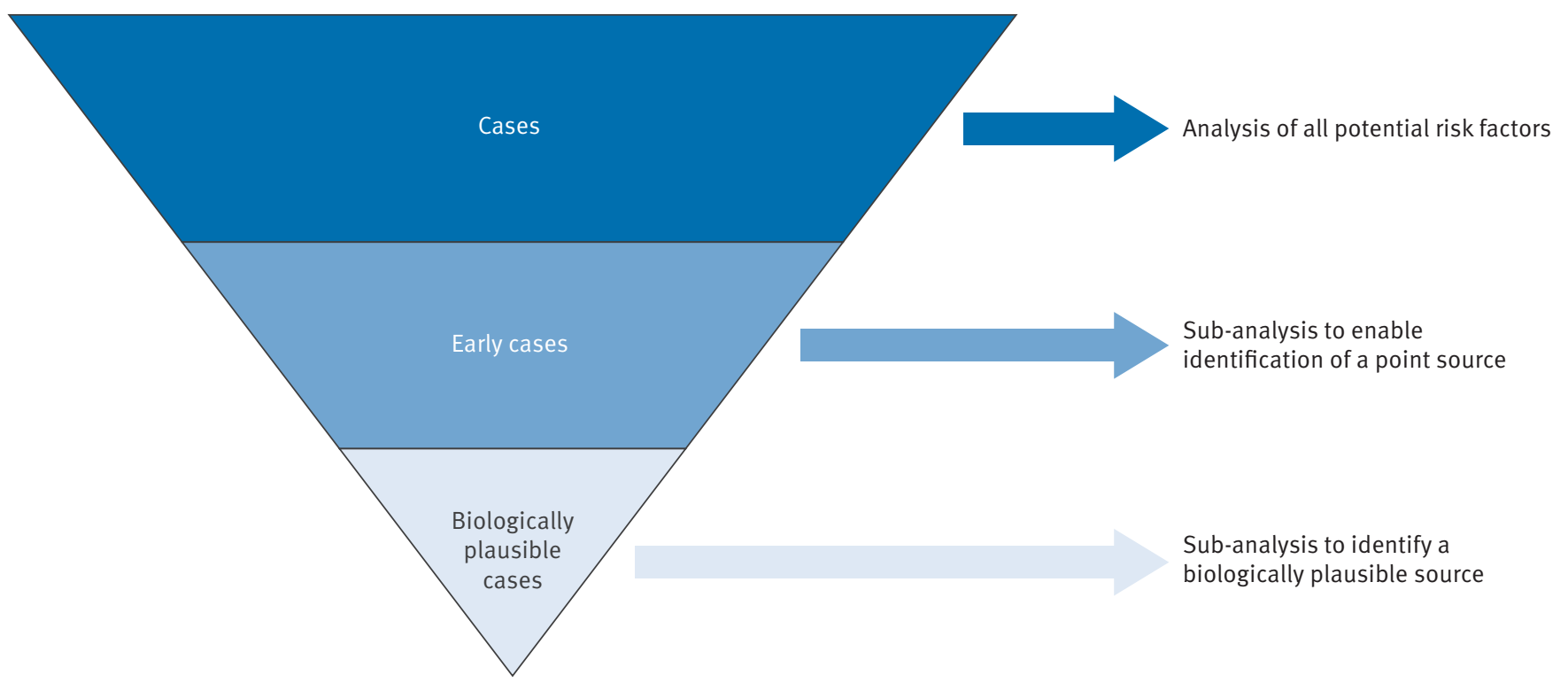

TABLE 1

Demographics of passengers of Coaches A and B, norovirus outbreak, February $2009(\mathrm{~N}=75)$

\begin{tabular}{|l|c|c|c|c|c|c|c|c|c|}
\hline & \multicolumn{4}{|c|}{ Male } & \multicolumn{3}{c|}{ Female } & \multicolumn{2}{c|}{ Total } \\
\cline { 2 - 10 } & Number & $\%$ & Mean age (years) & Number & $\%$ & Mean age (years) & Number & $\%$ & Mean age (years) \\
\hline Coach A & 15 & 44 & 67 & 19 & 56 & 62 & 34 & 100 & 64 \\
\hline Coach B & 12 & 29 & 63 & 29 & 71 & 66 & 41 & 100 & 65 \\
\hline
\end{tabular}


as a case showing symptoms within 72 hours after consumption of that specific food item or exposure to that specific risk factor (Figure 1). We analysed the data for Coach A and Coach B independently as the two passenger groups had not been in contact with each other. Although the travellers were served identical meals, the food items were not exactly the same for both trips. Therefore we considered it inadequate to analyse the data for the whole group together.

Univariate analysis was performed, calculating relative risks (RR) and 95\% confidence intervals (CI) for dichotomous individual exposures for becoming either a case or an early case in separate analysis. In addition, logistic univariate analysis was used to calculate point estimates including their $95 \% \mathrm{CI}$ for discrete variables of consumption frequencies. Multivariate analysis was performed, including the variables that were found to be significant during univariate analysis, with variables, such as age, treated as continuous where possible. The variables remained in the model if $p$ values were 0.10 , while the backward selection procedure was used. All analyses were stratified for Coach $A$ and B. Statistical analyses were performed using Microsoft Excel, SPSS version 15.0 and SAS version 9.1 for Windows.

\section{Environmental investigation and prevention measures}

On 12 February, we informed the local German public health office where the hotel was situated, and requested an environmental investigation in the hotel. The travel agency was provided with Dutch guidelines for hygiene measures in settings with successive passenger groups [9]. The passengers were provided with information, attached to the questionnaire, about norovirus infections and how to prevent further transmission.

\section{FIGURE 2}

Early and late cases by onset of symptoms, norovirus outbreak, February 2009 (N=32)

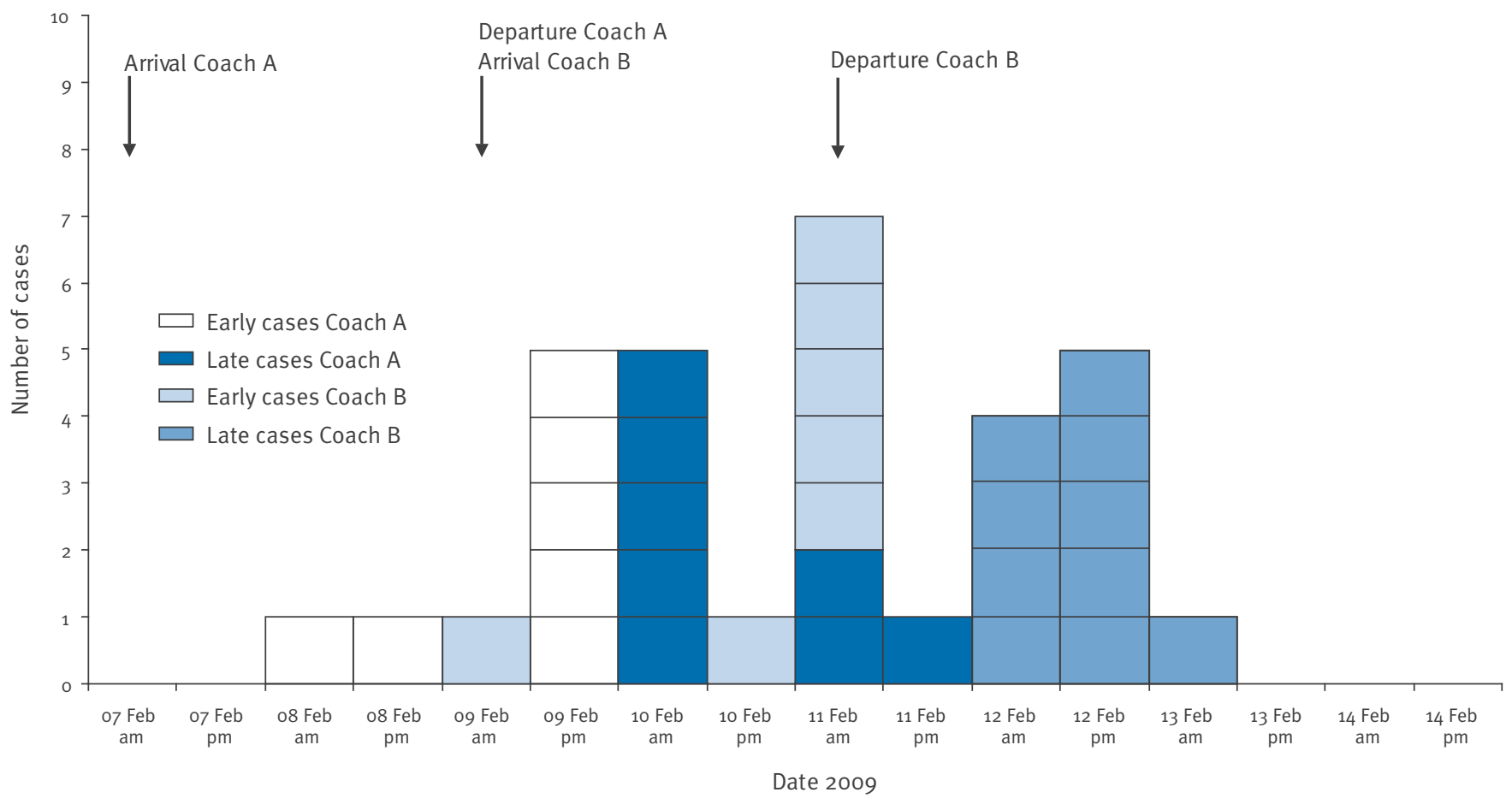

Date and hour of disease onset was available for 32 of 38 persons.

TABLE 2

Hotel menus with description of food items, norovirus outbreak, February 2009

\begin{tabular}{|c|c|c|c|c|c|c|c|}
\hline \multicolumn{8}{|l|}{ Day 1} \\
\hline Lunch & Soup & Bread & & & Juice & Coffee & Tea \\
\hline Dinner & Macaroni & Chicken/Turkey & Cabbage & & Juice & Coffee & Tea \\
\hline \multicolumn{8}{|c|}{ Days 2 and 3} \\
\hline Breakfast & Bread & Cheese & Egg & Jam & Juice & Coffee & Tea \\
\hline \multicolumn{8}{|l|}{ Day 2} \\
\hline Lunch & Chicken & Rice & Carrots & & Juice & Coffee & Tea \\
\hline Dinner & $\begin{array}{c}\text { Potatoes with } \\
\text { bacon }\end{array}$ & Pork chop & $\begin{array}{l}\text { Carrots in } \\
\text { sauce }\end{array}$ & & Juice & Coffee & Tea \\
\hline
\end{tabular}




\section{Results}

Laboratory Analysis

With four of four patient samples testing positive for norovirus in both ELISA and RT-PCR, this outbreak could be attributed to norovirus as the causative agent [10]. Sequencing of the RT-PCR fragments obtained from the four samples resulted in a full capsid sequence of ca. $1,600 \mathrm{nt}$, two partial capsid sequences of ca. $600 \mathrm{nt}$ and one partial capsid sequence of ca. $300 \mathrm{nt}$. All four sequences belonged to the norovirus genogroup II.4 variant $2006 \mathrm{~b}$, and had $100 \%$ identity.

\section{Epidemiological investigation}

Descriptive epidemiology

Of 87 listed passengers, 75 returned a filled questionnaire (response rate $86 \%$ ). Of these 75 passengers, $48(64 \%)$ were female and $27(36 \%)$ were male, with a mean age of 65 years for both sexes.

A total of 39 people met our case definition. One of these reported to have chronic diarrhoea and was therefore not considered to be a case, leaving 38 cases for analysis. The overall attack rate was 38 of $75(51 \%)$, with attack rates of 18 of $34(53 \%)$ and 20 of $41(49 \%)$ for Coach A and B, respectively. The attack rate for men and women in Coach A was $53 \%$ and $53 \%$, respectively (RR: 1.0, 95\% Cl: 0.5-1.9), and for Coach B $48 \%$ and $50 \%$, respectively (RR: $1.0,95 \% \mathrm{Cl}: 0.5-1.9$ ). As the attack rate for men and women was not different, we did not include sex as confounder in the data analysis.

Among the 38 cases, 28 persons had at least two episodes of diarrhoea and/or vomiting within 24 hours, and 10 persons reported one such episode, combined with nausea and watery diarrhoea, fever higher than 38 ${ }^{\circ} \mathrm{C}$, bowel cramps, headache and loss of appetite. The combinations of complaints were strongly indicative of a norovirus infection and therefore these people were included as cases. Nine of the 38 cases $(24 \%)$ reported slimy diarrhoea, $27(71 \%)$ reported watery diarrhoea, 13 (34\%) fever, 17 (45\%) stomach cramps, 29 (76\%) nausea and $32(84 \%)$ loss of appetite. Six passengers

\section{TABLE 3}

Analysis of food intake for all cases (A) and for early cases (B) in Coach A (N=34 $)$

A

\begin{tabular}{|l|c|c|c|c|c|c|c|c|c|}
\hline & \multicolumn{3}{|c|}{ Exposed } & \multicolumn{3}{|c|}{ Not exposed } \\
\hline Coach A (early and late cases) & III & Not ill & $\begin{array}{c}\text { AR } \\
\text { exposed }\end{array}$ & III & Not ill & $\begin{array}{c}\text { AR } \\
\text { unexposed }\end{array}$ & RR & $\begin{array}{c}95 \% \text { CI } \\
\text { exposed }\end{array}$ & $\begin{array}{c}\% \\
\text { eases }\end{array}$ \\
\hline Day 1 lunch & 18 & 14 & $56 \%$ & 0 & 2 & $0 \%$ & NA & NA & $100 \%$ \\
\hline Day 1 juice at lunch & 5 & 3 & $63 \%$ & 13 & 12 & $52 \%$ & 1.20 & $0.62-2.32$ & $28 \%$ \\
\hline Day 1 dinner & 17 & 15 & $53 \%$ & 1 & 1 & $50 \%$ & 1.06 & $0.26-4.41$ & $94 \%$ \\
\hline Day 1 juice at dinner & 3 & 4 & $43 \%$ & 14 & 11 & $56 \%$ & 0.77 & $0.30-1.93$ & $18 \%$ \\
\hline Day 2 breakfast & 17 & 13 & $57 \%$ & 1 & 3 & $25 \%$ & 2.27 & $\begin{array}{c}0.40- \\
12.73\end{array}$ & $94 \%$ \\
\hline Day 2 juice at breakfast & 11 & 7 & $61 \%$ & 7 & 8 & $47 \%$ & 1.31 & $0.68-2.52$ & $61 \%$ \\
\hline Day 2 lunch & 17 & 13 & $57 \%$ & 1 & 3 & $25 \%$ & 2.27 & $0.40-$ & $94 \%$ \\
\hline Day 2 juice at lunch & 1 & 3 & $25 \%$ & 17 & 11 & $61 \%$ & 0.41 & $0.07-2.31$ & $6 \%$ \\
\hline Day 2 dinner & 15 & 11 & $58 \%$ & 3 & 5 & $38 \%$ & 1.54 & $0.59-3.99$ & $83 \%$ \\
\hline Day 2 juice at dinner & 5 & 3 & $63 \%$ & 13 & 12 & $52 \%$ & 1.20 & $0.62-2.32$ & $28 \%$ \\
\hline Day 3 breakfast & 16 & 15 & $52 \%$ & 2 & 1 & $67 \%$ & 0.77 & $0.32-1.85$ & $89 \%$ \\
\hline Day 3 juice at breakfast & 7 & 6 & $54 \%$ & 11 & 9 & $55 \%$ & 0.98 & $0.52-1.86$ & $39 \%$ \\
\hline
\end{tabular}

B

\begin{tabular}{|l|c|c|c|c|c|c|c|c|c|}
\hline & \multicolumn{9}{|c|}{ Exposed } \\
\hline Coach A (early cases) & III & Not ill & $\begin{array}{c}\text { AR } \\
\text { exposed }\end{array}$ & III & Not ill & $\begin{array}{c}\text { AR } \\
\text { unexposed }\end{array}$ & RR & 95\% CI & $\begin{array}{c}\% \text { cases } \\
\text { exposed }\end{array}$ \\
\hline Day 1 juice at lunch & 4 & 4 & $50 \%$ & 6 & 17 & $26 \%$ & 1.92 & $0.72-5.09$ & $40 \%$ \\
\hline Day 1 dinner & 10 & 20 & $33 \%$ & 0 & 2 & $0 \%$ & NA & NA & $100 \%$ \\
\hline Day 1 juice at dinner & 1 & 4 & $20 \%$ & 9 & 16 & $36 \%$ & 0.56 & $0.09-3.46$ & $10 \%$ \\
\hline Day 2 breakfast & 10 & 18 & $36 \%$ & 0 & 4 & $0 \%$ & NA & NA & $100 \%$ \\
\hline Day 2 juice at breakfast & 7 & 10 & $41 \%$ & 3 & 11 & $21 \%$ & 1.92 & $0.61-6.09$ & $70 \%$ \\
\hline Day 2 juice at lunch & 1 & 3 & $25 \%$ & 9 & 17 & $35 \%$ & 0.72 & $0.12-4.27$ & $10 \%$ \\
\hline Day 2 dinner & 7 & 17 & $29 \%$ & 3 & 5 & $38 \%$ & 0.78 & $0.26-2.31$ & $100 \%$ \\
\hline Day 2 juice at dinner & 3 & 4 & $43 \%$ & 7 & 17 & $29 \%$ & 1.47 & $0.51-4.23$ & $30 \%$ \\
\hline Day 3 breakfast & 9 & 20 & $31 \%$ & 1 & 2 & $33 \%$ & 0.93 & $0.17-5.04$ & $90 \%$ \\
\hline Day 3 juice at breakfast & 5 & 7 & $42 \%$ & 5 & 14 & $26 \%$ & 1.58 & $0.58-4.33$ & $50 \%$ \\
\hline
\end{tabular}

AR: attack rate; $\mathrm{Cl}$ : confidence interval; NA: not applicable; RR: relative risk.

a Per food-item, the denominator varies. Answers were not given for all items on the questionnaires. 
(16\%) were admitted to hospital in the Netherlands. All recovered without sequelae.

The epidemic curve (Figure 2) shows the cases by date of onset of symptoms with a 12 hour interval, with distinction between the early and late cases for each of the two coaches. In both Coach A and Coach B, the initial cases occurred 24 hours after arrival in the hotel. The majority of the people in Coach B developed symptoms after departure from the hotel. A rapid drop in the incidence of new cases within the group of coach travellers is visible after arrival in the Netherlands when the group split up.

\section{Data analysis}

The menu served on day 1 , day 2 and day 3 are listed in Table 2 and were identical on both trips. We investigated the food items grouped together in meals as well as individual components of the meals for each coach trip separately, to see if there was an association between consumption and being a case in general or an early case.

Univariate analysis of the passengers from Coach A did not indicate a significant food-borne source for either cases in general (Table 3A) or early cases (Table 3B). However, illness of the early cases in Coach A was suggestive of being associated, albeit not significantly, with drinking juice on day 2 at breakfast (RR: 1.92; 95\% $\mathrm{Cl}$ : 0.6-6.1), day 2 at dinner (RR: $1.47 ; 95 \% \mathrm{Cl}: 0.5-4.2$ ) and day 3 at breakfast (RR: $1.58 ; 95 \% \mathrm{Cl}: 0.6-4 \cdot 3$ ).

As shown in Table 4, univariate analysis in the passengers from Coach B showed a significant association only with drinking juice. This is most obvious among the early cases (Table 4B), who had a strong association with drinking juice on day 1 at lunch (RR: 3.88 ; 95\% Cl: $1.3-11.7$ ), day 1 at dinner (RR: $5.45 ; 95 \% \mathrm{Cl}$ : 1.6-18.1) and a less strong association for day 2 at dinner (RR: 3.02; 95\% Cl: 1.0-9.4).

\section{TABLE 4}

Analysis of food intake for all cases (A) and for early cases (B) in Coach B (N=41 $)$

A

\begin{tabular}{|l|c|c|c|c|c|c|c|c|c|}
\hline & \multicolumn{9}{|c|}{ Exposed } \\
\hline Coach B (early and late cases) & III & Not ill & $\begin{array}{c}\text { AR } \\
\text { exposed }\end{array}$ & III & Not ill & AR unexposed & RR & $\begin{array}{c}\text { 95\% CI } \\
\begin{array}{l}\text { A cases } \\
\text { exposed }\end{array}\end{array}$ \\
\hline Day 1 lunch & 19 & 21 & $48 \%$ & 1 & 0 & $100 \%$ & 0.48 & $0.34-0.66$ & $95 \%$ \\
\hline Day 1 juice at lunch & 6 & 4 & $60 \%$ & 14 & 17 & $45 \%$ & 1.33 & $0.70-2.51$ & $30 \%$ \\
\hline Day 1 dinner & 10 & 1 & $91 \%$ & 10 & 20 & $33 \%$ & 2.73 & $1.59-4.68$ & $95 \%$ \\
\hline Day 1 juice at dinner & 18 & 18 & $50 \%$ & 2 & 3 & $40 \%$ & 1.25 & $0.41-3.84$ & $50 \%$ \\
\hline Day 2 breakfast & 14 & 11 & $56 \%$ & 6 & 10 & $38 \%$ & 1.49 & $0.73-3.07$ & $90 \%$ \\
\hline Day 2 juice at breakfast & 18 & 18 & $50 \%$ & 2 & 3 & $40 \%$ & 1.25 & $0.41-3.84$ & $70 \%$ \\
\hline Day 2 lunch & 6 & 3 & $67 \%$ & 14 & 18 & $44 \%$ & 1.52 & $0.83-2.79$ & $90 \%$ \\
\hline Day 2 juice at lunch & 9 & 3 & $75 \%$ & 11 & 18 & $38 \%$ & 1.98 & $1.12-3.49$ & $30 \%$ \\
\hline Day 2 dinner & 20 & 20 & $50 \%$ & 0 & 1 & $0 \%$ & NA & NA & $95 \%$ \\
\hline Day 2 juice at dinner & 9 & 8 & $53 \%$ & 11 & 13 & $46 \%$ & 1.16 & $0.62-2.16$ & $45 \%$ \\
\hline Day 3 breakfast & 19 & 20 & $49 \%$ & 1 & 1 & $50 \%$ & 0.97 & $0.23-4.04$ & $100 \%$ \\
\hline Day 3 juice at breakfast & 19 & 20 & $49 \%$ & 1 & 1 & $50 \%$ & 0.97 & $0.23-4.04$ & $45 \%$ \\
\hline
\end{tabular}

B

\begin{tabular}{|c|c|c|c|c|c|c|c|c|c|}
\hline & Exposed & & & $\begin{array}{c}\text { Not } \\
\text { exposed }\end{array}$ & & & & & \\
\hline Coach B (early cases) & III & Not ill & $\begin{array}{c}\text { AR } \\
\text { exposed }\end{array}$ & III & Not ill & $\begin{array}{c}\text { AR } \\
\text { unexposed }\end{array}$ & $\mathbf{R R}$ & $95 \% \mathrm{Cl}$ & $\begin{array}{l}\% \text { cases } \\
\text { exposed }\end{array}$ \\
\hline Day 1 juice at lunch & 5 & 5 & $50 \%$ & 4 & 27 & $13 \%$ & 3.88 & $1.28-11.70$ & $56 \%$ \\
\hline Day 1 dinner & 8 & 31 & $21 \%$ & 1 & 1 & $50 \%$ & 0.41 & $0.09-1.87$ & $89 \%$ \\
\hline Day 1 juice at dinner & 6 & 5 & $55 \%$ & 3 & 27 & $10 \%$ & 5.45 & $1.64-18.14$ & $67 \%$ \\
\hline Day 2 juice at breakfast & 8 & 17 & $32 \%$ & 1 & 15 & $6 \%$ & 5.12 & $0.71-37.15$ & $89 \%$ \\
\hline Day 2 juice at lunch & 3 & 6 & $33 \%$ & 6 & 26 & $19 \%$ & 1.78 & $0.55-5.74$ & $33 \%$ \\
\hline Day 2 dinner & 9 & 30 & $23 \%$ & 0 & 2 & $0 \%$ & NA & NA & $100 \%$ \\
\hline Day 2 juice at dinner & 5 & 7 & $42 \%$ & 4 & 25 & $14 \%$ & 3.02 & $0.98-9.35$ & $56 \%$ \\
\hline Day 3 breakfast & 9 & 31 & $23 \%$ & 0 & 1 & $0 \%$ & NA & NA & $100 \%$ \\
\hline Day 3 juice at breakfast & 5 & 12 & $29 \%$ & 4 & 20 & $17 \%$ & 1.76 & $0.55-5.62$ & $56 \%$ \\
\hline
\end{tabular}

AR: attack rate; $\mathrm{Cl}$ : confidence interval; NA: not applicable; RR: relative risk.

a Per food-item, the denominator varies. Answers were not given for all items on the questionnaires. 
When considering biological plausibility and dose of juice consumption associated with cases or early cases, we considered the number of meals at which juice was consumed each day, with a maximum of two for day 1 , three for day 2, and one for day 3. As the exact time of onset of symptoms was only known for 32 of 38 cases, we chose to use an onset of symptoms within four days after consumption to make sure the maximum incubation period of 72 hours was included. In Coach $A$, the risk from juice consumption increased per day, although non-significantly, with 1.4 (95\% Cl: $0.4-4.8$ ) for day $1,1.6$ (95\% Cl: $0.6-3.7$ ) for day 2 , and 2.0 (95\% Cl: $0.4-10.5$ ) for day 3 . In contrast, a decreasing and significant association was seen per day for coach B, with 5.0 (95\% Cl: 1.6-15.0) for day 1, 2.4 (95\% $\mathrm{Cl}: 1.0-5.6)$ for day 2 and $1.3(95 \% \mathrm{Cl}: 0.2-6.6)$ for day 3. Both groups experienced highest risk from consumption of juice served in a container on 9 February.

To assess whether visiting the toilet on board the coach during the return trip was a risk for becoming a case, biological plausibility was again considered, now by excluding the cases who had become ill before departure from the hotel. There appeared to be no significant risk of illness after using the onboard toilet (data not shown).

Despite low numbers, stratified multivariate logistic regression was performed to determine whether the univariate model could be improved. The backward selection model included the number of times juice was consumed on days 1, 2 and 3, lunches and dinners on days 1 and 2, breakfast on days 2 and 3, use of toilets in the hotel restaurant, coach and elsewhere during stops, and age in years. The analysis resulted in an invalid model which did not improve the univariate model. However, the results were consistent with univariate analysis, indicating a risk from juice consumption on 9 February in both groups.

\section{Prevention measures}

The German public health office performed an environmental health investigation in the hotel. Personal communication between the German and Dutch authorities revealed several critical points in the hygiene procedures. Both the MPHS Rotterdam-Rijnmond and the RIVM tried to obtain the results of the environmental investigation from the German public health office, but were informed that results of environmental investigations are not available to the human infectious disease unit due to regulations. Both coaches were thoroughly cleaned according to Dutch guidelines, and not directly used for successive passenger groups. No subsequent outbreaks or cases were notified from the travel agency. In addition, no additional secondary cases were reported after the groups split up in the Netherlands, breaking the transmission chain.

\section{Discussion and conclusion}

In this paper we show that consumption of juice may have contributed to an outbreak of norovirus infections among two cohorts of travellers following the same itinerary within a short period of time. We decided not to consider the two groups as one. Sub-analysis showed that the results were less conclusive, indicating that the two groups were different. The two groups of travellers had not been in contact with each other, did not share the same coach, and none of the cases had been in contact with persons who showed signs of gastroenteritis in the week preceding their journey. Moreover, onset of disease in all cases was after day 1 of the journey, suggesting a common point source of infection in each group.

Norovirus outbreaks are common and most often spread by person-to-person transmission. Because of their high attack rates, short incubation period and the high stability of the infectious agent in the environment, norovirus outbreaks are difficult to control. In addition, the identification of a common food-borne source is complicated because person-to-person transmission rapidly takes over after initial introduction of the virus through food. In order to be able to identify whether the virus had initially been introduced through food, we made a distinction between early and late cases, as early cases were more likely to be infected through food. This allowed us to identify an association between being an early case and drinking juice served in containers.

Although not all associations with consumption of juice served in a self-tap container were statistically significant and the $\mathrm{Cl}$ values included 1.0, we concluded that the results are nevertheless suggestive of an association, seeing as there was an RR of $\mathbf{3 . 0 2}$ for early cases in Coach B drinking juice at dinner on day 2, for whom the $\mathrm{Cl}$ shifted more from the right than to the left, also after correcting for non-lineairity of this relationship. Non-significance here may be an issue of small numbers. The results when considering biological plausibility, although not significant, did show an increase in risk per day in Coach $A$ and a decrease per day in Coach $B$ while considering the number of meals at which juice was consumed each day. We consider this to support the hypothesis of environmental contamination which most probably is linked to the use of the handle of the juice container.

It is generally difficult to determine whether food was contaminated during production or during preparation by infected food handlers [11]. Also in our study, contamination of the juice itself cannot be ruled out. It is not known whether the juice was tested for the presence of viruses. Virus detection in food involved in outbreaks, however, is generally complicated due to the low dose of viruses in food which may be below the detection level. Moreover, leftovers are rarely available for analysis. In particular, viral detection in fruit can be hampered by the presence of acid juices inhibiting the 
assay. Until validated assays are available, epidemiological evidence may assist in confirming a food-borne source [5]. However, the juice was served in a self-tap container during each meal buffet, making transmission through a contaminated environment possible. Also, our epidemiological evidence was indicative of transmission to the early cases through the contaminated juice container, with person-to-person transmission taking over for the later cases. Both groups were at highest risk on 9 February. It is possible that cleaning procedures in the hotel were different on days when tour groups changed due to tight time schedules, resulting in persisting environmental contamination. It is also possible that cleaning procedures after the arrival of the second coach reduced transmission and decreased the dose-response association of juice consumption for Coach B travellers. Unfortunately, we could not confirm this hypothesis since the report on the environmental investigation performed by the German public health office was not available for MPHS Rotterdam-Rijnmond.

Given the background prevalence of norovirus in the population and the presence of asymptomatic shedders, identifying norovirus as the causative agent of an outbreak of gastroenteritis is commonly based on a total of three samples, of which at least one should be positive for norovirus using RT-PCR [10]. In our study, only two samples in each group could be tested, but all were positive. Therefore, it is justified to assign the outbreak in both coaches to norovirus. Moreover, the strains detected were identical over a sequence length of 300 and $600 \mathrm{nt}$, strongly indicating that these outbreak strains were linked.

Often a case-definition of two or more episodes of diarrhoea and/or vomiting within 24 hours is used. We chose a case-definition of one or more episodes of diarrhoea and/or vomiting within 24 hours. As the 10 persons with only one such episode had also other symptoms which were strongly indicative of a norovirus infection, we consider our case definition justified.

Separating the early from the late cases appears to have been a successful method for distinguishing transmission modes, as described before [12]. An association with a food item was found in early cases, whereas person-to-person transmission or environmental transmission took over in the late cases. The late cases were probably predominantly infected through person-to-person spread of the virus in the coach on the way back to the Netherlands. Norovirus is known to spread easily from person to person in closed settings, as is seen for example on cruise ships [13]. After the passengers were back home in the Netherlands, the incidence fell rapidly and two days after departure from the hotel there were no new cases in either of the groups, suggesting that there was no exposure to a persisting common source or ill persons, and that providing the control guidelines contributed to limiting further spread.
Travelling has previously been described to be a risk factor for norovirus infection [14]. However, identification of the initial source in such outbreaks is difficult [15]. Outbreaks in travellers often involve several countries, which makes it difficult to collect all relevant data, a clear limitation of our study. In the Netherlands, cooperation between the various Public Health Services and the national Food and Consumer Product Safety Authority (VWA) is working well. If foodborne transmission is suspected, results of epidemiological and environmental investigations are jointly collected and shared. This is different from routine environmental control by the VWA. Different countries may have different surveillance systems, laws and regulations concerning privacy and sharing information. This interferes with international outbreak investigations and may profit from international guidelines and data sharing.

To our knowledge, this is the first time that consumption of juice served in containers is demonstrated to be a critical point in the hygiene procedure of hotels. Travel agencies and hotels should to be made aware that, once norovirus is introduced in a hotel setting, these containers need hygienic measures to limit further spread and prevent outbreaks in successive groups. Each outbreak investigation requires cooperation between actors in epidemiological, microbiological and environmental investigation, whether national or international. For a thorough investigation of outbreaks with international consequences, European guidance is needed regarding the collaboration of different authorities involved in cross-border outbreaks. In our opinion, the European Centre for Disease Prevention and Control is the body suited to provide such guidelines, in which it is important to address the potential lack of international comparability of laboratory data.

\section{Acknowledgements}

We would like to thank the local German Public Health Office (Gesundheitsamt) and the National Institute for Public Health and the Environment (RIVM) for their cooperation, Joukje Siebenga for the capsid sequences, the travel agency in providing the addresses of the travellers and the travellers themselves for their cooperation in returning the questionnaires.

\section{References}

1. Lopman BA, Reacher MH, Vipond IB, Sarangi J, Brown DW. Clinical manifestation of norovirus gastroenteritis in health care settings. Clin Infect Dis. 2004;39(3):318-24.

2. Friedman DS, Heisey-Grove D, Argyros F, Berl E, Nsubuga J, Stiles T, et al. An outbreak of norovirus gastroenteritis associated with wedding cakes. Epidemiol Infect. 2005;133(6):1057-63.

3. Godoy P, Izcara J, Bartolome R, Bach P, Escobar A, Pal M, et al. Toxiinfeccion alimentaria por Norovirus debida al consumo de bocadillos. [Outbreak of food-borne norovirus associated with the consumption of sandwiches]. Med Clin). 2005;124(5):161-4.

4. Teunis PF, Moe CL, Liu P, Miller SE, Lindesmith L, Baric RS, et al. Norwalk virus: how infectious is it? J Med Virol. 2008;80(8):1468-76. 
5. Verhoef L, Boxman I, Koopmans M. Viruses transmitted through the food-chain: a review of the latest developments. CAB Reviews. 2008;3:1-15.

6. Gotz H, Koopmans M, Bijlmer H. Een algoritme ter ondersteuning van de openbare gezondheidszorg bij uitbraken van gastro-enteritis [An algorithm to support diagnostics of gastroenteritis outbreaks in public health]. Ned Tijdschr Med Microbiol. 2008;16(2):11-15.

7. Svraka S, Duizer E, Vennema H, de Bruin E, van der Veer B, Dorresteijn $B$, et al. Etiological role of viruses in outbreaks of acute gastroenteritis in The Netherlands from 1994 through 2005. J Clin Microbiol. 2007;45(5):1389-94.

8. Siebenga JJ, Vennema H, Renckens B, de Bruin E, van der Veer B, Siezen RJ, et al. Epochal evolution of GGII.4 norovirus capsid proteins from 1995 to 2006. J Virol. 2007;81(18):9932-41.

9. National Institute for Public Health and the Environment (RIVM) [Internet]. Guideline Calicivirus (infection) . Bilthoven: RIVM. [Accessed May 2009]. Available from: http://www.rivm.nl/cib/ infectieziekten-A-Z/infectieziekten/calicivirus/index.jsp .

10. Duizer E, Pielaat A, Vennema H, Kroneman A, Koopmans M. Probabilities in norovirus outbreak diagnosis. J Clin Virol. 2007;40(1):38-42.

11. Verhoef L, Vennema H, Van Pelt W, Lees D, Boshuizen $H$, Henshilwood K, et al. Use of norovirus genotype profiles to differentiate origins of foodborne outbreaks. Emerg Infect Dis. 2010;16(4):617-24.

12. Götz H, Ekdahl K, Lindbäck J, de Jong B, Hedlund KO, Giesecke J. Clinical spectrum and transmission characteristics of infection with Norwalk-like virus: findings from a large community outbreak in Sweden. Clin Infect Dis. 2001;33(5):622-8.

13. Chimonas MA, Vaughan GH, Andre Z, Ames IT, Tarling GA, Beard $S$, et al. Passenger behaviors associated with norovirus infection on board a cruise ship--Alaska, May to June 2004. J Travel Med. 2008;15(3):177-83.

14. Rondy M, Koopmans M, Rotsaert C, Van Loon T, Beljaars B, Van Dijk G, et al. Norovirus disease associated with excess mortality and use of statins: a retrospective cohort study of an outbreak following a pilgrimage to Lourdes. Epidemiol Infect. Forthcoming 2010.

15. Verhoef L, Depoortere E, Boxman I, Duizer E, van Duynhoven Y, Harris J, et al. Emergence of new norovirus variants on spring cruise ships and prediction of winter epidemics. Emerg Infect Dis. 2008;14(2):238-43. 